\title{
Nanocellulose-Graphene Oxide Hybrid Aerogel for Water Purification
}

\author{
Jie Wei $^{a, 1}$, Shi-Han Gui ${ }^{a, 1}$, Jun-Hua Wu ${ }^{a, 2}$, Dan-Dan $\mathrm{Xu}^{1}$, Yun Sun ${ }^{1}$, Xiao-Ying Dong ${ }^{1, *}$, Yang-Yong Dai ${ }^{3, *}$, Yong-Feng Li ${ }^{1, *}$ \\ ${ }^{1}$ Department of Wood Science and Engineering, Forestry College, Shandong Agricultural University, Taian 271018, P.R. China \\ ${ }^{2}$ Materials Science and Engineering College, Beihua University, Jilin 132013, P.R. China \\ ${ }^{3}$ Department of Biological Science, College of Food Science and Engineering, Shandong Agricultural University, Taian 271018, P.R. China \\ ${ }^{a}$ These three authors contributed equally to this work
}

\begin{abstract}
Using green renewable resources to address the depletion of non-renewable resources and the pollution of industrial wastewater coincides with the sustainable development of human society. In this study, a hybrid aerogel is designed using nanocellulose and graphene oxide and served as a purifier to remove methylene blue (MB), congo red (CR) and waste oil in wastewater. The nanocellulose with abundant hydroxyl groups, a high aspect ratio $\sim 500$ and an average diameter of about $30 \mathrm{~nm}$ is uniformly distributed on the surface of graphene oxide sheets having a side length of about $1 \sim 3 \mu \mathrm{m}$, resulting the hybrid aerogel have a porosity of up to $99 \%$. When the mass ratio of nanocellulose and graphene oxide is $8: 2$, the hybrid aerogel reaches the highest adsorption capacity of $265.6 \mathrm{mg} / \mathrm{g}$ and $21.5 \mathrm{mg} / \mathrm{g}$ for MB and $\mathrm{CR}$, respectively. After further hydrophobic treatment, the hybrid aerogel shows excellent oil adsorption capacity up to $25.6 \mathrm{~g} / \mathrm{g}$, which is beneficial to oil/water separation. In a word, this strategy provides potential great-application of the nanocellulose in water purification.

Keywords: nanocellulose, graphene oxide, hybrid aerogel, water purification, oil/water separation, adsorption
\end{abstract}

${ }^{*}$ Correspondence to: Yong-Feng Li, Department of Wood Science and Engineering, Forestry College, Shandong Agricultural University, Taian 271018, China; E-mail: 1yf288@ hotmail.com

Xiao-Ying Dong, Department of Wood Science and Engineering, Forestry College, Shandong Agricultural University, Taian 271018, China; E-mail: dxiaoying1982@163.com

Yang-Yong Dai, Department of Biological Science, College of Food Science and Engineering, Shandong Agricultural University, Taian 271018, China; E-mail: dyyww@sdau.edu.cn

Received: November 9, 2018; Accepted: February 19, 2019; Published Online: February 26, 2019

Citation: Jie Wei, Shi-Han Gui, Jun-Hua Wu, Dan-Dan Xu, Yun Sun, Xiao-Ying Dong, Yang-Yong Dai, Yong-Feng Li, 2019. Nanocellulose-Graphene Oxide Hybrid Aerogel for Water Purification http://doi.org/10.26789/AEB.2019.01.003.

Copyright: Nanocellulose-Graphene Oxide Hybrid Aerogel for Water Purification.@ 2019 Jie Wei et al.. This is an Open Access article distributed under the terms of the Creative Commons Attribution-Noncommercial 4.0 International License, permitting all non-commercial use, distribution, and reproduction in any medium, provided the original work is properly cited and acknowledged.

\section{Introduction}

With the development of industry, dyestuff wastewater from factories (Dotto et al., 2013) and oil spills from sea transportation (Yang et al., 2014; Zhu et al., 2011) have seriously disturbed the balance of aquatic ecosystem and threatened the human health. The dyes in the wastewater are mostly composed of toxic organic materials, which could be decomposed by microorganisms via consuming oxygen in the water. However, this could cause the death of organisms due to lack of enough oxygen in the water and thus destroys the ecological balance of the water body (Nematollahzadeh et al., 2015; Nalan et al., 2015). While the oil spills contain highly toxic aromatic hydrocarbons, which could seriously damage human health due to their enrichment in food cycle (Yim et al., 2012). In the past few decades, many efforts have been devoted to developing various solutions to water pollution, including precipitation reduction (Kadam and Lee, 2015), chemical oxidation (Rosales et al., 2011; Salem et al., 2000), adsorption, membrane separation (Guo et al., 2016), biological treatment
(Punzi et al., 2015; Piccin et al., 2013). Among these methods, adsorption techniques is totally considered one of the most effective approaches for sewage purification owing to its easy operation, low cost, non-toxicity, and large adsorption capacity (Hu et al., 2017; Mittal et al., 2010).

Recently, researchers have explored a variety of adsorption materials, such as zeolite (Tsai et al., 2008), diatomite (Sun et al., 2017), activated carbon, chitosan (Ngah et al., 2011), sponge (Chen et al., 2018) and aerogel (Yang et al., 2016), to achieve the separation of dyes/oils from water. Wan et al. (Wan et al., 2017) reported the functional $\mathrm{MoS}_{2}$-coated melamine-formaldehyde $\left(\mathrm{MF} / \mathrm{MoS}_{2}\right)$ sponges with over $90 \%$ discoloration efficiency of removing methyl orange and methylene blue from water. Khosravi et al. synthesized a highly hydrophobic and highly oily polypyrrole/palmitic acid (PPy/PA) sponge by simple vapor deposition, polypyrrole polymerization and subsequent palmitic acid modification, which presents easily removal of different types of oils from sewage. However, these raw materials are non-renewable. 
Yogesh et al. prepared activated carbon with a specific surface area of $180.5 \mathrm{~m}^{2} / \mathrm{g}$ by pyrolyzing rice husk under $\mathrm{ZnCl}_{2}$ conditions, which could adsorb MB with capacity of $9.83 \mathrm{mg} / \mathrm{g}$. Namasivayam et al. studied activated carbon extracted from coir pith and the resulted activated carbon was able to adsorb CR with capacity of $6.7 \mathrm{mg} / \mathrm{g}$. Albadarin et al. explored the activation of lignin chitosan-extruded pellets by extrusion and thermal activation, which exhibits the largest adsorption of MB with capacity of $36.25 \mathrm{mg} / \mathrm{g}$. Although these raw materials are renewable, their adsorption capacity were quite poor. Therefore, developing renewable and highly adsorbent materials is very meaningful but challenging.

Nanocellulose, as a bright star in the nanomaterials, is one of the most ideal candidates for preparation of aerogels due to its high biocompatibility, renewability, abundance in source materials, high mechanical strength and high specific surface area (Zhuo et al., 2017; Zhuo et al., 2017; Zhu et al., 2016; Leitch et al., 2016 Geng et al., 2017; Wei et al., 2018; Dong et al., 2017). Graphene oxide regarded as the most popular and promising carbon nanomaterial is highly reported to explore the potential applications in water purification due to its huge specific surface area, high mechanical strength and easy functionality. Here, we design a hybrid aerogel by combining nanocellulose extracted from Amorpha fruticosa and graphene oxide for wastewater treatment via its high specific surface area and high porosity (Figure 1). When nanocellulose and graphene oxide are designed to form the hybrid aerogel with the optimal mass ratio of 8:2, it exhibits the highest adsorption of MB and CR from water with capacity of $265.6 \mathrm{mg} / \mathrm{g}$ and $21.5 \mathrm{mg} / \mathrm{g}$, respectively. And further simple hydrophobization endows the hybrid aerogel with functionality of oil-water separation. This work provides one strategy for the sustainable nanocellulose potentially applied in sewage purification.

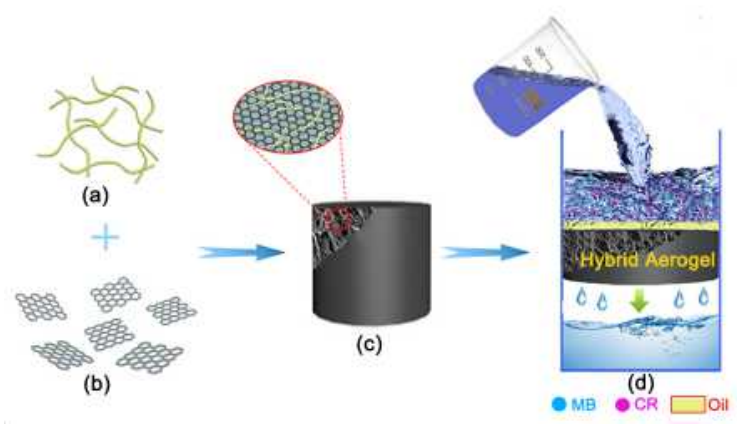

Figure 1. Schematic illustration to the fabrication of the hybrid aerogel and its adsorption of dyes (MB and CR) and oil from sewage. (a) nanocellulose; (b) graphene oxide; (c) the nanocellulose/graphene oxide hybrid aerogel; (d) separation of dyes and oil from water by adsorption of the hybrid aerogel.

\section{Materials and Methods}

\subsection{Materials}

Amorpha fruticosa, abtained from the suburb of Tai' an city in China, was crushed into 100-mesh powder for this experiment. 300-mesh graphite powder was purchased from Qingdao Hengli Graphite Co., Ltd. in China. Toluene, Anhydrous Ethanol, Potassium Hydroxide, Glacial Acetic Acid and Hydrogen Peroxide were all purchased from Tianjin Kaitong Chemical Reagent Co., Ltd. in China. Sodium Chlorite was provided by Shandong West Asia Chemical Industry Co., Ltd. in China. Phosphorus Pentoxide, Sodium Nitrate, Potassium Persulfate and Potassium Permanganate were purchased from Sichuan West Asia Chemical Reagent Co., Ltd. in China. Above all solvents were analytical reagent. Sulfuric Acid (98\%) and Hydrochloric Acid (37\%) were received from Jinan Daruiheng Chemical Co., Ltd. in China. $1 \mathrm{H}, 1 \mathrm{H}, 2 \mathrm{H}, 2 \mathrm{H}$-perfluorodecyltrimethoxysilane (17F) was obtained from Shanghai Hanhong Chemical Co., Ltd. in China. MB and CR were supplied by Tianjin Zhiyuan Chemical Reagent Co., Ltd. in China. Deionized water was used in all experiments.

\subsection{Preparation Procedures}

\subsubsection{Preparation of Nanocellulose}

The dried Amorpha fruticosa powder (100 mesh) was dissolved in phenylethyl-alcohol mixed solution at a volume ratio of 2:1 to remove the extracts (tannin, pigment, etc.). Then the extracted wood powder was treated by $1 \mathrm{wt} \%$ sodium chlorite for $6 \mathrm{~h}$ ( $1 \mathrm{~h}$ for each time, repeated 6 times) under acidic conditions ( $\mathrm{pH}$ at $4 \sim 5$ ) to remove lignin, followed by $5 \mathrm{wt} \%$ potassium hydroxide treatment for $2 \mathrm{~h}$ under heated water condition $\left(90^{\circ} \mathrm{C}\right)$ to remove hemicellulose. This process was repeated twice to obtain purified cellulose. After that, the purified cellulose fibers at concentration of $0.5 \mathrm{wt} \%$ in deionized water was treated by a high-pressure homogenizer (APV2000, SPX FLOW, Inc., Unna, Germany) for 20 times under conditions of the first valve of 600 bar and the second valve of 100 bar, followed by ultrasonication treatment in an ultrasonic generator (JY98-DN, Ningbo Xinzhi Biological Technology Inc., China) at $1000 \mathrm{~W}$ for $20 \mathrm{~min}$ to finally obtain the nanocellulose suspension.

\subsubsection{Preparation of the Hybrid Aerogel}

Firstly, graphene oxide was prepared via the modified Hummers method (Xu et al., 2013). A certain amount of graphene oxide was added into $0.5 \mathrm{wt} \%$ nanocellulose suspension (120 $\mathrm{ml}$ ) to form a mixture with 11 kinds of mass ratio (nanocellulose : graphene oxide, $10: 0,9: 1,8: 2,7: 3,6: 4$, $5: 5,4: 6,3: 7,2: 8,1: 9,0: 10$ ), which was then successively stirred at $14000 \mathrm{rpm}$ for $10 \mathrm{~min}$ and sonicated at $800 \mathrm{~W}$ for $10 \mathrm{~min}$ to obtain stable suspensions. Hereafter, the 11 kinds of mixtures were underwent a freezing process at $-55^{\circ} \mathrm{C}$ for $6 \mathrm{~h}$ in a freeze-drying machine (SCIENTZ-12N, 
Ningbo Xinzhi Biological Technology Inc., China), followed by a freeze-drying process at the same temperature for 48 $\mathrm{h}$ to obtain the final nanocellulose/graphene oxide hybrid aerogels with 11 kinds of mass ratios.

\subsection{Characterization and Performance Testing}

\subsubsection{Characterization}

Micromorphology of nanocellulose, graphene oxide and the nanocellulose/graphene oxide mixture were observed using scanning electron microscopy (FE-SEM, JEM-6610LV, JEOL USA Inc., Peabody, Massachusetts) and transmission electron microscopy (TEM, JEM-1400, JEOL USA Inc., Peabody, Massachusetts). Their chemical compositions were analyzed by X-ray photoelectron spectroscopy (XPS, Escalab 250Xi, Thermo Scientific Inc.,USA), X-ray diffractometer (XRD, D/max 2200, Rigaku Corporation), Fourier transform infrared spectrometer (FTIR, Thermo Nicolet Inc., USA), and Raman spectrometer (LabRAM HR800, Horiba jobin yvon Inc., France). The Bonding energies of XPS are referenced to the $\mathrm{C} 1 \mathrm{~s}$ neutral carbon peak at $284.8 \mathrm{eV}$. The XRD test parameters are as follows: the ray wavelength is 0.154 $\mathrm{nm}$, and the scanning angle ranges from $5^{\circ}$ to $60^{\circ}$, and the scanning speed is $4 \% \mathrm{~min}$, and the step length is $0.02^{\circ}$, and the tube voltage is $40 \mathrm{kV}$, and the tube current is $30 \mathrm{~mA}$. The data of FTIR and Raman spectrum are recorded in the range of $400-4000 \mathrm{~cm}^{-1}$ at a resolution of $4 \mathrm{~cm}^{-1}$. In addition, the excitation wavelength of $532 \mathrm{~nm}$ is generated by an Ar-ion laser.

\subsubsection{Evaluation of Density and Porosity of the Hybrid Aerogels}

The apparent density of the hybrid aerogel is calculated according to the following formula:

$$
\rho=\frac{m}{V}
$$

Where $\rho$ is the density of the hybrid aerogel, $m$ is the weight of the hybrid aerogel, and $\mathrm{v}$ is the volume of the hybrid aerogel.

The calculation formula of the porosity of the hybrid aerogel is as follows:

$$
P(\%)=100 \times\left\{1-\left(\frac{\rho \times \frac{n}{10}}{\rho_{a}}\right)-\left[\frac{\rho \times \frac{(10-n)}{10}}{\rho_{b}}\right]\right\}
$$

Where $P$ is the porosity of the hybrid aerogel, $\rho$ is the density of the hybrid aerogel, $\rho_{a}, \rho_{b}$ is the density of nanocellulose and graphene oxide, respectively; and $n$ is the fraction of nanocellulose accounting for the hybrid aerogel.

\subsubsection{Adsorption Capacity of the Hybrid Aerogel for Oils and Dyes}

(1) The hybrid aerogel was further treated with $17 \mathrm{~F}$ vapor to possess hydrophobicity. The hydrophobic hybrid aerogel was placed in a pump oil, which was floated on water surface. After adequate adsorption, the hybrid aerogel was removed and the oil adsorption capacity of the hybrid aerogel, $c(\mathrm{~g} / \mathrm{g})$, was calculated as follows:

$$
c=\frac{m_{1}-m_{0}}{m_{0}}
$$

where $c$ is the adsorption capacity, $m_{0}$ and $m_{1}$ is the weight of the hybrid aerogel before and after absorption, respectively. (2) The MB and CR standard solution was set to concentration $(\mathrm{C})$ of $1,2,4,6,8,10 \mathrm{mg} / \mathrm{mL}$, respectively, and the corresponding absorbance (Abs) was obtained at wavelength of $664 \mathrm{~nm}$ and $500 \mathrm{~nm}$, respectively. The concentrationabsorbance standard curve of MB (a) and CR (b) was plotted separately. The linear regression equation was as follows:

$$
\begin{aligned}
& A b s=0.1086 \times(C)+0.003 \quad R^{2}=0.9997 \quad(a) \\
& A b s=0.0118 \times(C)+0.0006 \quad R^{2}=0.9996 \quad(b)
\end{aligned}
$$
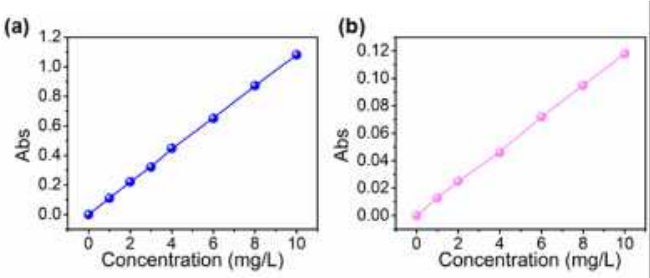

Figure 2. (a) the concentration-absorbance standard curve of MB; (b) the concentration-absorbance standard curve of CR

Firstly, the hybrid aerogel was immersed in $300 \mathrm{~mL} \mathrm{MB}$ solution (concentration of $10 \mathrm{mg} / \mathrm{L}$ ) or CR solution (concentration of $10 \mathrm{mg} / \mathrm{L}$ ), while another equal mass of hybrid aerogel was placed in $50 \mathrm{~mL}$ distilled water as control. Then, a beaker containing the hybrid aerogel and the above solution was placed on a cyclotron (120 r/min) and shaken for $24 \mathrm{~h}$ at room temperature, after that the hybrid aerogel was not taken out until the adsorption equilibrium was reached. The MB or CR solution was centrifuged to obtain a supernatant. The concentration of the MB or CR solution was determined by a UV-Vis spectrophotometer (722N.S, Shanghai Youke instrument Inc., China) at $664 \mathrm{~nm}$ or $540 \mathrm{~nm}$. The adsorption value $(Q)$ of the hybrid aerogel for $\mathrm{MB} / \mathrm{CR}$ was calculated as follows:

$$
Q=\frac{\left(c_{0}-c_{e}\right) v}{1000 \times m}
$$

Where $Q(\mathrm{mg} / \mathrm{g})$ indicates the adsorbed value of $1 \mathrm{~g}$ hybrid aerogel for $\mathrm{MB}$ or $\mathrm{CR}$ when the adsorption reaches equilibrium. $c_{0}$ and $c_{e}$ was the initial concentration of the $\mathrm{MB}$ or $\mathrm{CR}$ solution and the equilibrium concentration of the MB or $\mathrm{CR}$ solution, respectively. $v$ and $m$ was the volume of the MB or CR solution and the mass of the hybrid aerogel, respectively.

\section{Results and Discussion}

The nanocellulose suspension presents stable state, indicating that the nanocellulose well dispersed in water (Figure 


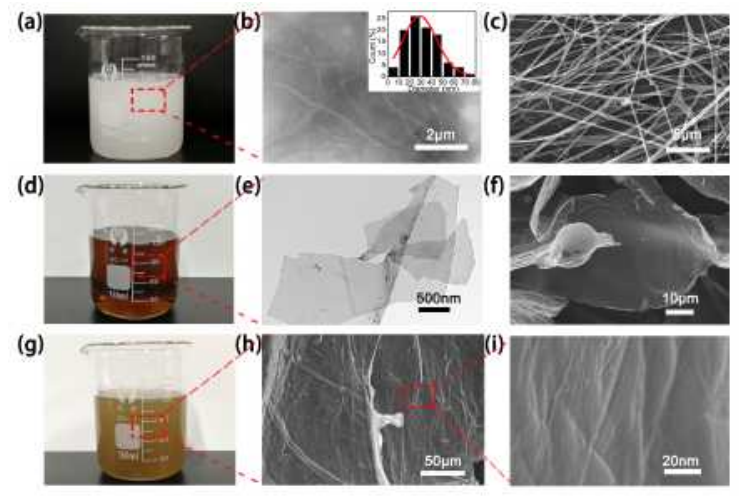

Figure 3. Microscopic characterization of nanocellulose, graphene oxide, nanocellulose/graphene oxide mixture. (a) digital photo of nanocellulose suspension; (b) TEM image of nanocellulose and statistic analysis of the diameter of nanocellulose (inset); (c) SEM image of nanocellulose; (d) digital photo of graphene oxide suspension; (e) TEM image of graphene oxide; (f) SEM image of graphene oxide; (g) digital photo of nanocellulose/graphene oxide mixture; (h) SEM image of nanocellulose/graphene oxide; (i) is the magnified SEM image of (h).

3a). The TEM image shows fine and uniform structure of nanocellulose with average diameter of $\sim 30 \mathrm{~nm}$ and length of over ten micrometers (Figure $3 \mathrm{~b}$ and inset), indicating the nanocellulose possessing high aspect ratios of $\sim 500$. The SEM image (Figure 3c) shows the nanocelluloses in uniform filament state and intertwine with each other, forming a threedimensional network structure, which might be beneficial to form high porous aerogel to remove pollutants from water. GO dispersed in deionized water presents brown color (Figure $3 \mathrm{~d}$ ). TEM image shows that the graphene oxide presents transparent thin sheet with side length of $1 \sim 3 \mathrm{~mm}$, indicating large specific surface (Figure 3e). The SEM image shows GO sheet with similar microstructure, further proving the large specific surface of the derived GO (Figure 3f). The nanocellulose/graphene oxide mixture presents pale yellow without any delamination and precipitation, suggestting that nanocellulose and graphene oxide forms stable mixture (Figure $3 \mathrm{~g}$ ). SEM images shows that the nanocellulose filaments are tightly attached onto the graphene oxide lamellae (Figure $3 \mathrm{~h}, \mathrm{i}$ ), which benefits to the further preparation of the hybrid aerogel.

After the freeze-drying process, the nanocellulose/graphene oxide mixture forms the hybrid aerogel. Theoretically, the aerogel has a highly porous structure due to the water removal via the direct sublimation process (Figure 4a). Figure 4d describes and proves the porous structure of the aerogel. Figure $4 \mathrm{~b}$ presents that the hybrid aerogel in dark brown color could well stand on a green leaf, suggesting quite lower density. Figure $4 \mathrm{c}$ and $4 \mathrm{e}$ proves the ultralight feature and quite lower density of the hybrid aerogel, respectively. The hybrid aerogel achieves an ultralight density of $\sim 2.151 \mathrm{mg} / \mathrm{cm}^{3}$, which is about 14 times lower than the density of the polyurethane sponge reported in the literature (Figure 4c). Additionly, the aerogel presents porosity over $99.88 \%$, which is also higher than that of the polyurethane sponge (Figure 4e) (Zhou et al., 2013). (a)
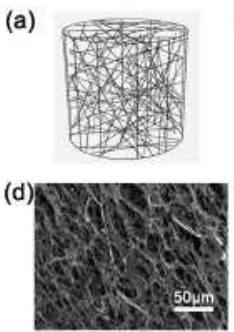

(g)

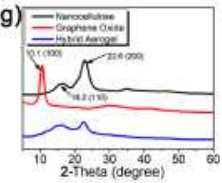

(b)
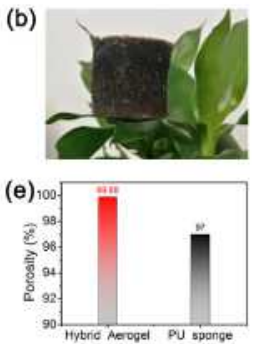

(h)

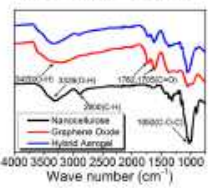

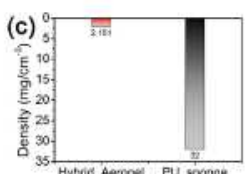

(f)

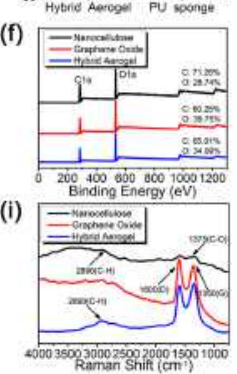

Figure 4. Density/porosity analysis of the hybrid aerogel and the chemical characterization of nanocellulose, graphene oxide and the hybrid aerogel. (a) Diagram illustration of the hybrid aerogel; (b) digital photo of the hybrid aerogel; (c) density comparison of the hybrid aerogel and polyurethane (PU) sponge; (d) SEM image of the hybrid aerogel; (e) porosity comparison of the hybrid aerogel and PU sponge; (f) XPS spectra of nanocellulose, graphene oxide, and the hybrid aerogel; (g) XRD curves of nanocellulose, graphene oxide, and the hybrid aerogel; (h) FTIR spectra of nanocellulose, graphene oxide, and the hybrid aerogel; (i) Raman spectra of nanocellulose, graphene oxide, and the hybrid aerogel.

In order to further understand the bonding mode of nanocellulose and graphene oxide in the hybrid aerogel, XPS, XRD, FTIR, and Raman spectroscopy were utilized to investigate their chemical composition information. Figure $4 \mathrm{f}$ shows that the hybrid aerogel consists of $\mathrm{C}$ and O element with weight percentage of $65.01 \%$ and $34.99 \%$, respectively. With the addition of graphene oxide, the content of $\mathrm{O}$ elements in the aerogel increases from 28.74 wt $\%$ of pure cellulose to 34.99 wt $\%$ of hybrid aerogel, which means the hybridization of graphene oxide and nanocellulose. Figure $4 \mathrm{~g}$ describes that the nanocellulose has traditional diffraction peaks at $2 \theta=16.2^{\circ}$ and $2 \theta=22.6^{\circ}$, corresponding to the plane of (110) and (200), respectively, which suggests that the nanocellulose still retained the crystal I structure during mechanical treatment. Graphene oxide shows obvious diffraction peak at $2 \theta=10.1^{\circ}$, while the hybrid aerogel contains each the characteristic diffraction peak of nanocellulose or graphene oxide, suggesting mainly physical interaction without obvious chemical interaction between nanocellulose and graphene oxide. Figure $4 \mathrm{~h}$ presents that the characteristic peaks of the hybrid aerogel were the overlap of the characteristic peaks of nanocellulose and graphene oxide, including the stretching vibration peak at $2890 \mathrm{~cm}^{-1}(\mathrm{C}-\mathrm{H})$ and $3326 \mathrm{~cm}^{-1}(\mathrm{O}-\mathrm{H})$ in the nanocellulose, and stretching vibration peak at $1782 \mathrm{~cm}^{-1}$ and $1705 \mathrm{~cm}^{-1}(\mathrm{C}=\mathrm{O})$ in the graphene oxide. This proved that the nanocellulose and graphene oxide were physically combined in the hybrid aerogel. The results presented in Raman spectroscopy are consistent with that in FTIR spectrum. Figure $4 i$ proves that two distinct characteristic peaks (the $\mathrm{D}$ band at $1350 \mathrm{~cm}^{-1}$ and the $\mathrm{G}$ band at 1600 $\mathrm{cm}^{-1}$ ) of graphene oxide and the hydroxyl absorption peak of the nanocellulose were the same as those exhibited in 
hybrid aerogel, suggesting physical interaction between the two nanomaterials.

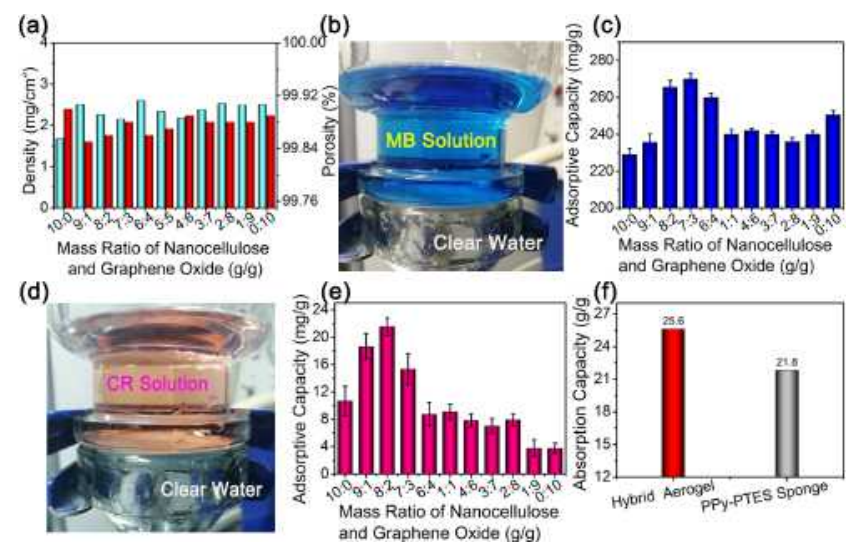

Figure 5. Density/porosity and adsorption properties of the hybrid aerogels with different mass ratios. (a) density/porosity of hybrid aerogels with different mass ratios; (b) digital photo of adsorption of MB by the hybrid aerogel; (c) adsorption capacity of hybrid aerogels with different mass ratios for MB; (d) digital photo of adsorption of CR by the hybrid aerogel; (e) adsorption capacity of hybrid aerogels with different mass ratios for CR; (f) oil-water separation capacity of the hybrid aerogel.

To determine the optimum ratio of nanocellulose and graphene oxide, 11 different mass ratios of the hybrid aerogels were designed and examined by comparing their adsorption capacity for MB and CR. As we all know, the higher the porosity and the larger the specific surface area of the hybrid aerogel, the stronger the adsorption capacity for dyes and oils in the sewage. Actually, the porosity of the hybrid aerogel provides extremely large volume for the entrance of liquids and the specific surface area provides more physical interaction between liquids like dye molecules or lipid molecules and the cell wall of the hybrid aerogel via their active contact sites. Figure 5a shows that all the hybrid aerogels with different mass ratios have lower density of less than $2.7 \mathrm{mg} / \mathrm{cm}^{-3}$ and higher porosity of up to $99.8 \%$, which is beneficial to the purification of sewage by the hybrid aerogel. The adsorption capacity of the hybrid aerogel was evaluated by extracting $\mathrm{MB} / \mathrm{CR}$ liquid through a $4 \mathrm{~cm}$-diameter hybrid aerogel in a self-made filtration device. The blue MB solution became colorless when infiltrating through the hybrid aerogel (Figure 5b). The hybrid aerogels with 11 mass ratios presented different adsorption capacity for MB. Among them, when the mass ratio of nanocellulose and graphene oxide was 7:3, the hybrid aerogel achieves the highest adsorption capacity of up to $265.6 \mathrm{mg} / \mathrm{g}$ for MB (Figure 5c). Excitingly, the hybrid aerogel also presents adsorption behavior for CR (from original red color to colorless) (Figure 5d). It is found that the hybrid aerogel obtains the largest adsorption capacity of $21.5 \mathrm{mg} / \mathrm{g}$ for CR when the mass ratio of nanocellulose and graphene oxide was 8:2 (Figure 5e). Considering the comprehensive adsorption effect of the hybrid aerogel on $\mathrm{MB}$ and CR, we finally determines 8:2 as the optimal mass ratio of nanocellulose and graphene oxide for water purification. More interestingly, the hybrid aerogel after hydrophobization exhibits excellent pump oil adsorption capacity, up to 25.6 times weight of the original hybrid aerogel (Figure 5f), which is higher than that of the oil adsorption material reported in the literature (Zhou et al., 2013; Phanthong et al., 2018; Lin et al., 2015).

\section{Conclusion}

In this work, we successfully design and prepare a hybrid aerogel with ultralight density $\left(2.151 \mathrm{mg} / \mathrm{cm}^{-3}\right)$ and superhigh porosity $(99.8 \%)$ using nanocellulose and graphene oxide via a simple freeze-drying process. Chemical analysis show that the nanocellulose and graphene oxide are physically combined in the hybrid aerogel, which could exert the advantages of both high aspect ratio and high specific surface area of nanocellulose and graphene oxide. The hybrid aerogel presents excellent adsorption behavior on separation of dyes and waste oils from wastewater. When the mass ratio of nanocellulose and graphene oxide is $7: 3$, the hybrid aerogel shows the highest adsorption capacity of up to 265.6 $\mathrm{mg} / \mathrm{g}$ for MB. And when the mass ratio of nanocellulose and graphene oxide reaches 8:2, the hybrid aerogel exhibits the highest adsorption capacity of $21.5 \mathrm{mg} / \mathrm{g}$ towards CR. Additionly, the hydrophobic hybrid aerogel presents higher oil adsorption capacity of up to 25.6 times its own weight. Such hybrid aerogel could be regarded as one of the most promising adsorption materials for sewage purification.

\section{Acknowledgments}

We acknowledge the financial supports from the Natural Science Foundation of Shandong Province, Doctoral Branch (Grant. No. ZR2017BC042), and Innovation Project of Shandong Provincial Forestry Science and Technology (Grant. No. LYCX10-2018-50), and Key Special Foundation for the National Key Research and Development Program of China (Grant. No. 2016YFD0600704), and National Natural Science Foundation of China (Grant. No. 31700497; 31471619), and "13th Five-Year" Science and Technology Project of Jilin Provincial Education Department (Grant. No. JJKH20170038KJ).

\section{Conflict of Interest}

The authors declare no competing financial interest.

\section{Author contributions}

Jie Wei, Shihan Gui, Junhua Wu and Yongfeng Li designed the experiment. Jie Wei and Shihan Gui performed the whole experiments. Junhua Wu helped to analyze the characterizations. Dandan $\mathrm{Xu}$ and Yun Sun drew the figures. Jie Wei, Shihan Gui and Xiaoying Dong carried out performance evaluations. Jie Wei, Yangyong Dai and Yongfeng Li wrote the paper. Everybody comments on the final manuscript. ${ }^{a} \mathrm{Jie}$ 
Wei, Shihan Gui and Junhua Wu contributed equally to this work.

\section{References}

Albadarin, A.B., Collins, M.N., Naushad, M., Shirazian, S., Walker, G., Mangwandi, C., 2017. Activated lignin-chitosan extruded blends for efficient adsorption of methylene blue. Chemical Engineering Journal, 307, 264-272. http://www.doi.org/10.1016/j.cej.2016.08.089

Chen, T., Shi, P., Zhang, J., Li, Y., Duan, T., Dai, L., Wang, L., Yu, X., Zhu, W., 2018. Natural polymer konjac glucomannan mediated assembly of graphene oxide as versatile sponges for water pollution control. Carbohydrate Polymers, 202, 425-433. http://www.doi.org/10.1016/j.carbpol.2018.08.133

Dong, X.Y., Zhuo, X., Wei, J., Zhang, G., Li, Y.F., 2017. Woodbased nanocomposite derived by in-situ formation of organicinorganic hybrid polymer within wood via a sol-gel method. ACS Applied Materials \& Interfaces, 9(10), 9070-9078. http://www.doi.org/10.1021/acsami.7b01174

Dotto, G.L., Moura, J.M., Cadaval, T.R.S., Pinto, L.A.A., 2013. Application of chitosan films for the removal of food dyes from aqueous solutions by adsorption. Chemical Engineering Journal, 214(4), 8-16. http://www.doi.org/10.1016/j.cej.2012.10.027

Geng, B., Wang, H., Wu, S., Ru, J., Tong, C., Chen, Y., Liu, H., Wu, S., Liu, X., 2017. Surface-tailored nanocellulose aerogels with thiol-functional moieties for highly efficient and selective removal of $\mathrm{Hg}$ (II) ions from water. ACS Sustainable Chemistry \& Engineering, 5(12), 11715-11726. http://www.doi.org/10.1021/acssuschemeng.7b03188

Guo, Y., Wang, X., Hu, P., Peng, X., 2016. ZIF-8 coated polyvinylidenefluoride (PVDF) hollow fiber for highly efficient separation of small dye molecules. Applied Materials Today, 5, 103-110.

http://www.doi.org/10.1016/j.apmt.2016.07.007

Hu, J., Deng., W., Chen, D., 2017. Ceria hollow spheres as an adsorbent for efficient removal of acid dye. ACS Sustainable Chemistry \& Engineering, 5(4), 3570-3582. http://www.doi.org/10.1021/acssuschemeng.7b00396

Kadam, A.A., Lee, D.S., 2015. Glutaraldehyde cross-linked magnetic chitosan nanocomposites: Reduction precipitation synthesis, characterization, and application for removal of hazardous textile dyes. Bioresource Technology, 193, 563-567. http://www.doi.org/10.1016/j.biortech.2015.06.148

Khosravi, M., Azizian, S., 2015. Synthesis of a novel highly oleophilic and highly hydrophobic sponge for rapid oil spill cleanup. ACS Applied Materials \& Interfaces, 7(45), 2532625333. http://www.doi.org/10.1021/acsami.5b07504

Leitch, M.E., Li, C., Ikkala, O., Mauter, M.S., Lowry, G.V., 2016. Bacterial nanocellulose aerogel membranes: novel high-porosity materials for membrane distillation. Environmental Science \& Technology Letters, 3(3), 85-91. http://www.doi.org/10.1021/acs.estlett.6b00030

Lin, R., Li, A., Zheng, T., Lu, L., Cao, Y., 2015. Hydrophobic and flexible cellulose aerogel as an efficient, green and reusable oil sorbent. RSC Advances, 5, 82027-82033.

http://www.doi.org/10.1039/C5RA15194E
Mittal, A., Mittal, J., Malviya, A., Kaur, D., Gupta, V.K., 2010. Adsorption of hazardous dye crystal violet from wastewater by waste materials. Journal of Colloid \& Interface Science, 343(2), 463-473.

http://www.doi.org/Adsorption of hazardous dye crystal violet from wastewater by waste materials

Nalan, O.S.K., Asl1, C., Tamer, U., Turgay, T., 2015. Microalgae immobilized by nanofibrous web for removal of reactive dyes from wastewater. Industrial \& Engineering Chemistry Research, 54(21), 5802-5809. http://www.doi.org/10.1021/acs.iecr.5b01033

Namasivayam, C., Kavitha, D., 2002. Removal of congo red from water by adsorption onto activated carbon prepared from coir pith, an agricultural solid waste. Dyes \& Pigments, 54(1), 47-58. http://www.doi.org/10.1016/S0143-7208(02)00025-6

Nematollahzadeh, A., Shojaei, A., Karimi, M., 2015. Chemically modified organic/inorganic nanoporous composite particles for the adsorption of reactive black 5 from aqueous solution. Reactive \& Functional Polymers, 86(26), 7-15. http://www.doi.org/10.1016/j.reactfunctpolym.2014.11.001

Ngah, W.S.W., Teong, L.C., Hanafiah, M.A.K.M., 2011. Adsorption of dyes and heavy metal ions by chitosan composites: A review. Carbohydrate Polymers, 83(4), 1446-1456. http://www.doi.org/10.1016/j.carbpol.2010.11.004

Phanthong, P., Reubroycharoen, P., Kongparakul, S., Samart, C., Wang, Z., Hao, X., Abudula, A., Guan, G., 2018. Fabrication and evaluation of nanocellulose sponge for oil/water separation. Carbohydrate Polymers, 190, 184-189. http://www.doi.org/10.1016/j.carbpol.2018.02.066

Piccin, J.S., Feris, L.A., Cooper, M., Gutterres, M., 2013. Dye adsorption by leather waste: mechanism diffusion, nature studies, and thermodynamic data. Journal of Chemical \& Engineering Data, 58, 873-882. http://www.doi.org/10.1021/je301076n

Punzi, M., Anbalagan, A., Aragão, B.R., Svensson, B.M., Jonstrup, M., Mattiasson, B., 2015. Degradation of a textile azo dye using biological treatment followed by photo-Fenton oxidation: evaluation of toxicity and microbial community structure. Chemical Engineering Journal, 270, 290-299. http://www.doi.org/10.1016/j.cej.2015.02.042

Rosales, E., Pazos, M., Sanroman, M., 2011. Comparative efficiencies of the decolourisation of leather dyes by enzymatic and electrochemical treatments. Desalination, 278, 312-317. http://www.doi.org/10.1016/j.desal.2011.05.041

Salem, I.A., El-Maazawi, M.S., 2000. Kinetics and mechanism of color removal of methylene blue with hydrogen peroxide catalyzed by some supported alumina surfaces. Chemosphere, 41(8), 1173-1180. http://www.doi.org/10.1016/S0045-6535(00)00009-6

Sun, Z., Yao, G., Liu, M., Zheng, S., 2017. In situ synthesis of magnetic $\mathrm{MnFe}_{2} \mathrm{O}_{4}$ /diatomite nanocomposite adsorbent and its efficient removal of cationic dyes. Journal of the Taiwan Institute of Chemical Engineers, 71, 501-509. http://www.doi.org/10.1016/j.jtice.2016.12.013

Tsai, C.K., Liao, C.Y., Wang, H.P., Chien, Y.C., Jou, C.J., 2008. Pyrolysis of spill oils adsorbed on zeolites with product oils recycling. Marine Pollution Bulletin, 57(6), 895-898. http://www.doi.org/10.1016/j.marpolbul.2008.02.041

Wan, Z., Li, D., Jiao, Y., Ouyang, X., Chang, L., Wang, X., 2017. Bifunctional MoS2 coated melamine-formaldehyde sponges 
for efficientoil-water separation and water-soluble dye removal. Applied Materials Today, 9, 551-559.

http://www.doi.org/10.1016/j.apmt.2017.09.013

Wei, J., Zhang, G., Dong, J., Wang, H., Guo, Y., Zhuo, X., Li, C., Liang, H., Gu, S., Li, C.H., Dong, X.Y., Li, Y.F., 2018. Facile, scalable spray-coating of stable emulsion for transparent self-cleaning surface of cellulose-based materials. ACS Sustainable Chemistry \& Engineering, 6 (9), 11335-11344. http://www.doi.org/10.1021/acssuschemeng.8b00962

Xu, Z., Sun, H., Zhao, X., Gao, C., 2013. Ultrastrong fibers assembled from giant graphene oxide sheets. Advanced Materials, 25(2), 188-193.

http://www.doi.org/10.1002/adma.201203448

Yang, Y., Deng, Y., Tong, Z., Wang, C., 2014. Renewable ligninbased xerogels with self-cleaning properties and superhydrophobicity. ACS Sustainable Chemistry \& Engineering, 2(7), 1729-1733.

http://www.doi.org/10.1021/acssuschemeng.7b00440

Yang, H., Sheikhi, A., Tg, V.D.V., 2016. Reusable green aerogels from crosslinked hairy nanocrystalline cellulose and modified chitosan for dye removal. Langmuir, 32(45), 1177111779. http://www.doi.org/10.1021/acs.langmuir.6b03084

Yim, U.H., Kim, M., Ha, S.Y., Ha, S., Kim, S., Shim, W., 2012. Oil spill environmental forensics: the Hebei Spirit oil spill case. Environmental Science \& Technology, 46(12), 6431-6437. http://www.doi.org/10.1021/es3004156

Sharma, Y.C., 2010. Optimization of parameters for adsorption of methylene blue on a low-cost activated carbon. Journal of Chemical \& Engineering Data, 55(1), 435-439. http://www.doi.org/10.1021/je900408s

Zhou, X., Zhang, Z., Xu, X., Men, X., Zhu, X., 2013. Facile fabrication of superhydrophobic sponge with selective absorption and collection of oil from water. Industrial \& Engineering Chemistry Research, 52(27), 9411-9416. http://www.doi.org/10.1021/ie400942t

Zhu, H., Qiu, S., Jiang, W., Wu, D., Zhang, C., 2011. Evaluation of electrospun polyvinyl chloride/polystyrene fibers as sorbent materials for oil spill cleanup. Environmental Science \& Technology, 45(10), 4527-4531. http://www.doi.org/10.1016/j.aca.2018.02.016

Zhu, H., Luo, W., Ciesielski, P.N., Fang, Z.Q., Zhu, J.Y., Henriksson.G., Himmel.M.E., Hu.L.B., 2016. Wood-eerived materials for green electronics, biological devices, and energy applications. Chemical Reviews, 116(16), 9305-9374. http://www.doi.org/10.1021/acs.chemrev.6b00225

Zhuo, X., Liu, C., Pan, R., Dong, X.Y., Li, Y.F., 2017. Nanocellulose mechanically isolated from Amorpha fruticosa Linn. ACS Sustainable Chemistry \& Engineering, 5(5), 4414-4420. http://www.doi.org/10.1021/acssuschemeng.7b00478

Zhuo, X., Wei, J., Xu, J., Pan, R., Zhang, G., Guo, Y.L., Dong, X.Y., Long, L., Li, Y.F., 2017. Nanocellulose isolation from Amorpha fruticose by an enzyme-assisted pretreatment. Applied Environmental Biotechnology, 2(1), 34-39. http://www.doi.org/10.26789/AEB.2017.01.005 